University of Nebraska - Lincoln

DigitalCommons@University of Nebraska - Lincoln

7-1-1998

\title{
Iron and nickel surface and interface anisotropies
}

\author{
Ralph Skomski \\ University of Nebraska-Lincoln, rskomski2@unl.edu \\ D. Sander \\ Max-Planck-Institut fur Mikrostrukturphysik, Weinberg 2, 06120 Halle, Germany \\ C. Schmidthals \\ Max-Planck-Institut fur Mikrostrukturphysik, Weinberg 2, 06120 Halle, Germany \\ Axel Enders \\ Max-Planck-Institut fur Mikrostrukturphysik, a.enders@me.com \\ J. Kirschner \\ Max-Planck-Institut fur Mikrostrukturphysik
}

Follow this and additional works at: https://digitalcommons.unl.edu/physicsskomski

Part of the Physics Commons

Skomski, Ralph; Sander, D.; Schmidthals, C.; Enders, Axel; and Kirschner, J., "Iron and nickel surface and interface anisotropies" (1998). Ralph Skomski Publications. 12.

https://digitalcommons.unl.edu/physicsskomski/12

This Article is brought to you for free and open access by the Research Papers in Physics and Astronomy at DigitalCommons@University of Nebraska - Lincoln. It has been accepted for inclusion in Ralph Skomski Publications by an authorized administrator of DigitalCommons@University of Nebraska - Lincoln. 


\title{
Iron and nickel surface and interface anisotropies
}

\author{
R. Skomski, ${ }^{*}$ D. Sander, C. Schmidthals, A. Enders, and J. Kirschner \\ Max-Planck-Institut für Mikrostrukturphysik, Weinberg 2, 06120 Halle, Germany \\ *Present address: Department of Physics and Astronomy, University of Nebraska, Lincoln NE 68588
}

\begin{abstract}
The anisotropy of fec (111) Fe/Ni and $\mathrm{Fe} / \mathrm{Ni} / \mathrm{Fe}$ layers is investigated. The films exhibit an oscillating behavior of the preferential magnetization direction depending on whether the surface layer is $\mathrm{Ni}$ or $\mathrm{Fe}$. Anisotropy fields are obtained from polar and perpendicular Kerr hysteresis loops and yield a comparatively small perpendicular $\mathrm{Fe} / \mathrm{Ni}$ interface anisotropy of about $0.15 \mathrm{~mJ} / \mathrm{m}^{2}$. The real-space origin of the interface anisotropy is the interlayer hybridization of the $y z, z x$, and $z^{2}$ orbitals.
\end{abstract}

Index terms - interface anisotropy, ultrathin films, Kerr hysteresis

\section{INTRODUCTION}

Magnetic anisotropy, that is the dependence of the magnetic energy on the magnetization direction, is a property of major scientific and technological interest. In ultrathin films, surface and interface anisotropies are non-negligible and often dominate the bulk contributions [1]- [5]. A key question is the explanation and prediction of the magnetic anisotropy from the atomic structure and the d-band filling of the atoms involved. Fe/Ni films are interesting because they serve as a tool to study interface anisotropies between different $3 \mathrm{~d}$ elements [6], [7]. As discussed in Ref. [6], by thermal evaporation $(\mathrm{MBE})$ it is possible to produce well characterized films where intermixing (interface alloying) between $\mathrm{Fe}$ and $\mathrm{Ni}$ is negligible.

Key features of the metallic $3 d$ anisotropy are the comparatively weak spin-orbit coupling, the itinerant character of the magnetic electrons, and the nontrivial involvement of subband densities of states (DOS). Basically, on has to diagonalize the oneelectron band-structure Hamiltonian

$$
\mathcal{H}=-\frac{\hbar^{2}}{2 \mathrm{~m}} \frac{\partial^{2}}{\partial \mathbf{r}^{2}}+\mathrm{V}(\mathbf{r})+\lambda \hat{\mathbf{L}} \cdot \hat{\mathbf{s}}
$$

The last term, where $\hat{\mathbf{C}}=-\mathrm{i}(\mathbf{r} \times \partial / \partial \mathbf{r})$ and $\hat{\mathbf{s}}=\hat{\boldsymbol{\sigma}} / 2$ are (dimensionless) orbital angular momentum and spin operators, respectively, describes the spin-orbit interaction. For the late $3 \mathrm{~d}$ elements, $\lambda \approx 40 \mathrm{meV}$.

The atomic spin-orbit coupling, that is the magnetostatic interaction of the spin with the electron's own orbital moment, is isotropic, because there is no unique quantization axis in free atoms. Aniso- tropy is caused by the spin-dependent one-electron potential $\mathrm{V}$, which obeys the symmetry of the magnet and affects the motion of the electrons [8]- [10]. In $3 \mathrm{~d}$ metals, the leading mechanism is anisotropic interatomic hopping.

A common numerical approach is to calculate the anisotropy from perturbative band-structure expressions such as

$$
\delta E(\theta)=-\lambda^{2} \sum_{\mathrm{o}, \mathrm{u}} \frac{\langle\mathrm{o}|\hat{\mathbf{L}} \cdot \mathbf{s}(\theta)| u\rangle<\mathrm{u}|\hat{\mathbf{L}} \cdot \hat{\mathbf{s}}(\theta)| \mathrm{o}\rangle}{\mathrm{E}_{\mathrm{u}}-\mathrm{E}_{\mathrm{O}}}
$$

where $o$ and $u$ denote occupied and unoccupied band-structure levels [1], [3], [11]-[13]. However, those demanding and time-consuming calculations are at the expense of physical transparency. Here we will discuss the problem of $3 \mathrm{~d}$ interface anisotropy from a more qualitative point of view.

\section{EXPERIMENTAL RESULTS}

A variety of ultrathin fcc (111) $\mathrm{Fe} / \mathrm{Ni}$ and $\mathrm{Fe} / \mathrm{Ni} / \mathrm{Fe}$ layers on a W(110) substrate have been deposited by thermal evaporation (MBE). After deposition, magneto-optical Kerr (MOKE) angle and ellipticity measurements are used to obtain polar and longitudinal hysteresis loops. Figure 1 shows typical film profiles and hysteresis loops for in-plane and perpendicular magnetization directions. In this example 9 mono-

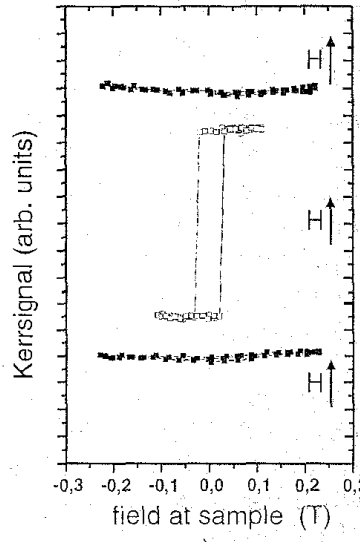

a)

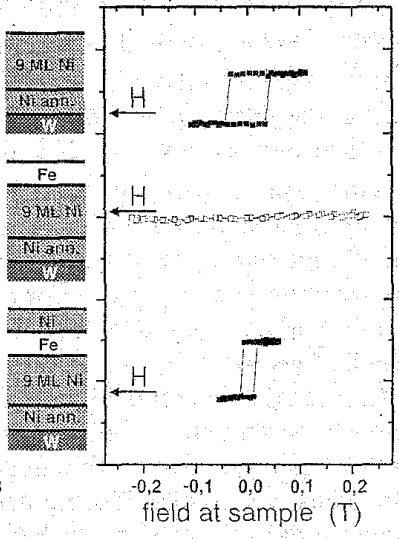

b)
Fig. 1. Layer profiles and Kerr magnetization curves of Fe/Ni magnets: (a) perpendicular and (b) in-plane. 
layers (ML) of Ni have been deposited on $1 \mathrm{ML} \mathrm{Ni}$ on W(110), which was annealed at $900 \mathrm{~K}$ for several minutes [6].

The almost square loop measured parallel to the film shows that the preferential magnetization direction lies in the film plane. An additional atomic layer of Fe leads to a transition from easy-plane to perpendicular anisotropy, as indicated by the square loop in the perpendicular direction. The important point is that a cap layer of Ni reverses this transition and turns the preferential magnetization direction back in the film plane. In other words, for a fairly wide range of layer thicknesses the preferential magnetization direction is in-plane or perpendicular, depending on whether the respective surface layer consists of $\mathrm{Ni}$ or $\mathrm{Fe}$.

Starting from relations such as $\mu_{\mathrm{O}} \mathrm{H}_{\mathrm{A}} \Sigma_{\mathrm{i}} \mathrm{M}_{\mathrm{i}} \mathrm{t}_{\mathrm{i}} \approx$ $2 \sum \mathrm{K}_{\mathrm{s}}-\mu_{\mathrm{O}} \sum_{\mathrm{i}} \mathrm{M}_{\mathrm{i}} 2 \mathrm{t}_{\mathrm{i}}$ and analyzing the anisotropy fields $\left(\mathrm{H}_{\mathrm{A}}\right)$ in terms of the $\mathrm{Fe}$ and Ni layer thicknesses $t_{i}$ the $\mathrm{Fe} / \mathrm{Ni}$ interface anisotropy is investigated. The slopes of the magnetization curves and the reorientation transitions $\left(\mathrm{H}_{\mathrm{A}}=0\right)$ yield the anisotropy estimate $\mathrm{K}_{\mathrm{Fe} / \mathrm{Ni}}=0.15 \pm .10 \mathrm{~mJ} / \mathrm{m}^{2}$, whereas the difference $\mathrm{K}_{\mathrm{Fe}} / \mathrm{UHV}-\mathrm{K}_{\mathrm{Ni} / \mathrm{UHV}}$ is of order 0.6 $\mathrm{mJ} / \mathrm{m}^{2}$. Thus, the $\mathrm{Fe} / \mathrm{Ni}$ interface gives rise to a comparatively small perpendicular anisotropy.

\section{THEORETICAL INTERPRETATION}

From the Schrödinger equation for spherical potentials one obtains five atomic $3 d$ wave functions characterized by quantum numbers $n=3,1=2$, and $\mathrm{m}$. A particular feature of magnetic anisotropy is the individual involvement of the five $3 \mathrm{~d}$ sublevels, whereas metallic $3 \mathrm{~d}$ moments can be estimated from the total density of states. There exist two sets of atomic wave functions [14]. The $\phi$ dependence of real wave functions $|\mu\rangle$, such as $|\mathrm{xy}\rangle$, is given by factors $\sin \left(\mathrm{m} \phi-\mathrm{m} \phi_{\mathrm{o}}\right)$, whereas complex wave functions $\mid \pm m>$ exhibit an $\exp ( \pm \mathrm{im} \phi)$ dependence. Each set of wave functions is orthonormal and complete, but averages such as $\mathrm{L}_{\mathrm{Z}}=\left\langle\psi\left|\hat{\mathrm{L}}_{\mathrm{Z}}\right| \psi\right\rangle$ are zero and nonzero for real and complex wave functions, respectively. This means that real wave functions, which are also known as quenched orbitals or standing waves, do not contribute to the anisotropy.

The real or complex nature of atomic wave functions is determined by the competition between crystal-field and spin-orbit interactions. Real wave functions can reduce their energy by adapting themselves to the crystal environment, whereas complex running-wave orbitals are favorable from the point of view of spin-orbit interaction. In 3d magnets, the hopping and crystal-field interactions dominate, and the wave functions are largely quenched. The spin-orbit coupling merely acts as a perturbation and yields a small admixture of running-wave character and some anisotropy.

\section{A. Band-filling dependence}

Due to quenching, one has to interpret itinerant anisotropy in terms of real $3 \mathrm{~d}$ orbitals. There are two types of orbitals: the 'in-plane' $x y$ and $x^{2}-y^{2}$ orbitals, and the 'out-of-plane' $y z, z x$, and $z^{2}$ orbitals (Fig. 2). In monolayers and at surfaces, the interatomic hopping between in-plane orbitals is more pronounced than that between out-of-plane orbitals. As a consequence, the $x y$ and $x^{2}-y^{2}$ subband widths $\mathrm{W}_{\mu}$ are largest and the states at the top and at the bottom of the $\uparrow$ and $\downarrow$ subbands have in-plane character. In lowest order [15], this rule determines the subband fillings as a function of the number $n$ of $3 d$ electrons.

The total anisotropy is obtained by adding all pair contributions [1], [3]. In general, both $\uparrow$ and $\downarrow$ subbands need to be considered, although the leading interaction is that between $\downarrow$ electrons. The spinorbit interaction between two $\downarrow$ levels $\mid \mu>$ and $|\mu\rangle$ yields a perpendicular anisotropy contribution for $|\mathrm{m}|=|\mathrm{m}|$ but an easy-plane contribution for $\left|\mathrm{m}^{\prime}\right|=$ $|\mathrm{m} \pm 1|$. Since spin-space rotations by an angle $\theta=\pi$ corresponds to real-space rotations by an angle $\theta / 2$ $=\pi / 2$, the reverse is true when the two levels are occupied by electrons of opposite spin [1].

For example, the relation $\mathrm{L}_{z}=-\mathrm{i} \partial / \partial \phi$ means that $x y$ and $x^{2}-y^{2}$ states can reduce their energy by spin orbit coupling if the spin is perpendicular to the surface. Since the states at the top of the band have $x y$ and $x^{2}-y^{2}$ character, nearly filled bands $(n>9.5)$ yield perpendicular anisotropy. Unfortunately, $\mathrm{Ni}-$ $\mathrm{Cu}$ surface and interface anisotropies are difficult to measure due to an unfavorable signal-noise ratio.
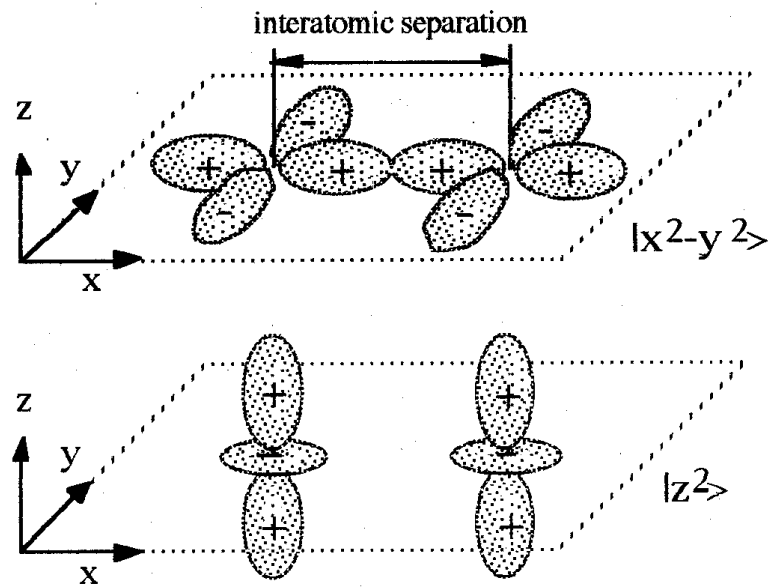

Fig. 2. Overlap of $3 d$ orbitals in monolayer films: 
As a crude rule, $\mathrm{Ni}$ and $\mathrm{Co}$ exhibit easy-plane surface anisotropies, whereas the anisotropy contribution of $\mathrm{Fe}$ is often, but not always, perpendicular [1], [4], [5], [15]. In the case of Ni, the anisotropy is determined by the strong easy-plane contribution of the out-of-plane $\downarrow$ bands. Note that a similar dependence is obtained from the quasimolecular diatomic pair model [3].

\section{B. Interface anisotropy}

The only structural information considered until now is that the number of in-plane neighbors exceeds that of out-of-plane neighbors. This gives a fair description of the band-filling behavior of the anisotropy in terms of nearest-neighbor numbers and lattice constants. In particular, according to (2) anisotropy scales as $1 / \mathrm{W}_{\mu}$ and anisotropy is largest for narrow subbands. Since $\mathrm{W}_{\mu}$ increases with the interatomic overlap of the $\mu$ orbitals, surface and interface out-of-plane bands are wider than in monolayers, and the magnitude of surface and interface anisotropies is comparatively small.

To distinguish interfaces from monolayers and surfaces we have to take into account the chemistry of the involved atoms [1], [16] (Fig. 3(a)). If there was no chemical difference between adjacent layers, then the equal widths of the in-plane and out-ofplane bands would yield zero interface anisotropy [17]. However, in reality the different d-band fillings break the cubic symmetry.

A simple approximation is the rigid-band model, where undistorted $\mathrm{Fe}$ and $\mathrm{Ni}$ bands are filled until a common Fermi level is reached. Up to secondary ndependent changes in the densities of states $D(E)$, the rigid-band model yields zero interface anisotropy (Fig. 3(b)). However, from 3d alloys it is known that the rigid-band model leads to unphysically large, unscreened charge transfers. The selfconsistent readjustment of the local potentials [18] yields skewed densities of states such as those shown in Fig. 3(c). As a consequence, changes in the subband fillings are comparatively small and adjacent $\mathrm{Fe}$ and $\mathrm{Ni}$ layers keep some free-standing

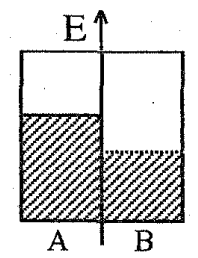

(a) isolated atoms

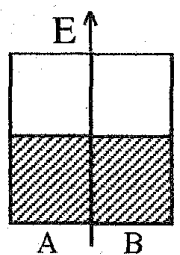

(b) charge transfer

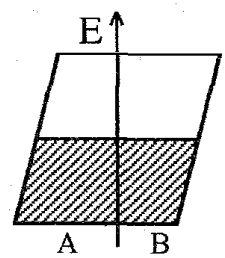

(c) charge neutrality
Fig. 3 Local densities of states and band filling for small differences $n_{A}-n_{B}$ : (b) rigid-band model and (c) selfconsistent skewing. standing character. Note, however, that the ndependent shift of the $\uparrow$ and $\downarrow$ subband centers of gravity $[1],[16]$ modifies this mechanism, so that the present explanation is rather qualitative.

\section{CONCLUSIONS}

In conclusion, we have investigated the anisotropy of $\mathrm{Fe} / \mathrm{Ni}$ interfaces. Kerr measurements yield an interface anisotropy of order $0.15 \mathrm{~mJ} / \mathrm{m}^{2}$. Analyzing $3 \mathrm{~d}$ subbands in terms of nearest neighbor geometries yields the rule that the magnitudes of $3 d$ surface and interface anisotropies are smaller than the magnitudes of free-standing monolayers. However, for interfaces containing late $3 \mathrm{~d}$ elements this mechanism yields zero anisotropy. In this case, the leading mechanism is the hybridization between overlapping $3 \mathrm{~d}$ subbands.

\section{REFERENCES}

11] J. A. C. Bland and B. Heinrich, Eds, Ultrathin Mmagnetic Structures I, Berlin: Springer, 1994.

[2] U. Gradmann, in: Handbook of Magnetic Materials, vol. 7, K. H. J. Buschow, Ed., Elsevier: Amsterdam 1993, pp. 1-95.

[3] D.-Sh. Wang, R.-Q. Wu, and A. J. Freeman, Phys. Rev. $B$, vol. 47, 1993, pp. 14932-14947.

[4] B. Heinrich and J. F. Cochran, Adv. Phys, vol. 42, 1993, pp. 523-639.

[5] M. T. Johnson, P. J. H. Bloemen, F. J. A. den Broeder, and J. J. de Vries, Rep. Prog. Phys., vol. 59, 1996, pp. 1409-1458.

[6] D. Sander, A. Enders, C. Schmidthals, J. Kirschner, H. L. Johnston, C. S. Arnold, and D. Venus, J. Appl. Phys., vol. 81, 1997, pp. 4702-4704.

[7] E. Colombo, O. Donzelli, G. B. Fratucello, and F. Ronconi, J. Magn. Magn. Mater., vol. 104-107, 1992 , pp. 1857-1858.

[8] C. J. Ballhausen, Ligand Field Theory, New York: McGraw-Hill, 1962.

[9] M. T. Hutchings, Solid State Phys, vol. 16, 1964, pp. 227-273.

[10] R. Skomski, IEEE Trans, Magn., vol. 32, 1996, pp. 4794-4796.

[11] H. Brooks, Phys. Rev., vol. 58, 1940, pp. 909-918.

[12] E. M. Kondorski and E. Straube, Zh. Eksp. Teor. Fiz., vol. 63, 1972, pp. 356-365.

[13] J. G. Gay and R. Richter, Phys. Rev. Lett., vol. 56, 1986, pp. 2728-2731.

[14] L. Pauling and E. B. Wilson, Introduction to Quantum Mechanics, McGraw-Hill: New York, 1935.

[15] R. Skomski, these proceedings.

[16] D.-Sh. Wang, R.-Q. Wu, and A. J. Freeman, J. Magn. Magn. Mater., vol. 129, 1994, p. 237.

[17] Here we neglect the small fourth-order cubic bulk anisotropy.

[18] A. P. Sutton, Electronic Structure of Materials, University Press: Oxford, 1993. 